\title{
Threshold Selection for CT Angiography Shaded Surface Display of the Renal Arteries
}

\author{
Ethan J. Halpern, Richard J. Wechsler, and Dennis DiCampli
}

\begin{abstract}
The purpose of this study was to define objective criteria to calculate a tissue segmentation threshold for shaded surface display (SSD) rendering of the renal arteries with computed tomography angiography. Contrast-enhanced spiral CT scans were obtained through the renal arteries of nine patients. Six sets of SSD images were rendered for each patient with lower threshold values ranging from 80 to $\mathbf{1 3 0}$ Hounsfield units (HU) by increments of $10 \mathrm{HU}$. Visceral organ enhancement was measured in the aorta, liver, spleen, pancreas, and kidney. The segmentation threshold for each patient was determined by evaluation of the SSD images alone as well as by comparison with conventional arteriograms. The ideal threshold, as shown by comparison with conventional arteriography, was better correlated with a threshold value selected by qualitative evaluation of SSD images alone $\left(r_{\mathrm{s}}=.42\right)$, than with measured enhancement in visceral organs $\left(r_{\mathrm{s}}=-.289\right.$ to .009$)$. The degree of stenosis was overestimated in a single renal artery (1 of 18) because of an inappropriate threshold selected by evaluation of the SSD images alone. In comparison with a segmentation threshold calculated from measured enhancement of visceral organs, a segmentation threshold selected by qualitative evaluation of the resulting SSD images is more likely to approximate the ideal threshold. Given the subjective nature of such threshold selection, further evaluation is warranted to determine whether threshold selection may result in inaccurate grading of stenosis.
\end{abstract}

Copyright 11995 by W.B. Saunders Company

7 TWO CATEGORIES of image-rendering techniques, surface and volume rendering, have been applied to create three-dimensional (3D) images from computed tomography (CT) data. ${ }^{1,2,3}$ A surface-rendering technique such a shaded surface display (SSD) requires tissue segmentation to define the surface of interest. ${ }^{4}$ Volume-rendering techniques such as maximum intensity projection (MIP) and ray sum may be implemented with or without tissue segmentation. ${ }^{4}$

Tissue segmentation is the classification of each volume element (voxel) within a volumet-

From the Department of Radiology, Jefferson Medical College, Thomas Jefferson University Hospital, Philadelphia, PA.

Address reprint requests to Ethan J. Halpern, $M D$, Department of Radiology, Thomas Jefferson University, $132 \mathrm{~S}$ 10th St, Philadelphia, PA 19107-5244.

Copyright $\odot 1995$ by W.B. Saunders Company

0897-1889/95/0803-0006\$3.0010 ric data set as to tissue type. Osseous structures may be identified by the high CT numbers characteristic of calcium. For CT angiography, each voxel is classified as vessel or nonvessel. Because vascular structures are enhanced with intravenous contrast, they may be identified by thresholding. An optimal threshold for CT angiography will include arterial structures and exclude the surrounding soft tissues. However, arterial enhancement is a dynamic process that varies over time in a given patient, and from patient to patient. Furthermore, the surrounding tissues also enhance to varying degrees. Thus, the "optimal" threshold for segmentation of arteries from adjacent soft tissues varies with injection site and rate as well as with circulation times and cardiac function.

Several early investigators of spiral CT have described promising results with CT angiography in general, ${ }^{5,6}$ and with $3 \mathrm{D}$ SSD in particular. ${ }^{7,8}$ However, one recent study suggested that the severity of renal artery stenosis (RAS) was most accurately depicted by axial and multiplanar reformatted images rather than by $3 \mathrm{D}$ renderings. ${ }^{9}$ Another recent study advocated MIP imaging over SSD for grading RAS ${ }^{10}$ Both of these studies noted changes in the SSD appearance of the renal arteries as a function of the segmentation threshold. When the threshold was increased, stenoses appeared more significant. The threshold for tissue segmentation in these studies was based upon either the CT density of enhanced soft tissues on transaxial images or upon a subjective evaluation of the resulting SSD images. No empirically validated, quantitative method has been described to determine tissue segmentation thresholds for SSD CT angiography.

Ideally, the threshold selected for CT angiography should yield a SSD image that matches the conventional arteriogram in terms of vessel caliber and degree of stenosis. The goal of the present study was to determine whether a quantitative technique based upon enhancement of visceral structures might provide a better estimate of the ideal threshold than an estimate based upon subjective evaluation of SSD images. 


\section{MATERIALS AND METHODS}

Seventeen consecutive CT angiograms performed at our institution during a 1-year period were retrospectively identified. These studies were ordered either to detect the presence of RAS or as part of the preoperative work up for aortic aneurysm repair. Conventional arteriographic correlation was available for six patients with suspected RAS and three patients with known aortic aneurysms. This group of nine patients comprises our study sample. The original group of 17 patients consisted of nine men and eight women with a mean age of 65 (range, 38 to 79 ). The study sample consisted of three men and six women with a mean age of 67 (range, 38 to 79 ).

All CT imaging was performed with a $9800 \mathrm{HiSpeed}$ Advantage spiral CT scanner (General Electric, Milwaukee, WI). Scans were obtained during the arterial phase after an intravenous injection of Omnipaque 300 (Sanof Winthrop, New York, NY). A 25-mL test bolus was injected at $3.5 \mathrm{~mL} / \mathrm{s}$ in each patient to measure the time delay between intravenous contrast administration and peak abdominal aortic enhancement at the level of the renal arteries. A 30-second helical scan was then obtained during a single breath hold at the time of peak aortic enhancement after a peripheral intravenous injection of $110 \mathrm{~mL}$ of Omnipaque 300 at $3.5 \mathrm{~mL} / \mathrm{s}$.

Patients with suspected RAS were evaluated with 3-mm collimation, allowing a 9-cm scan in craniocaudal dimension. Aortic aneurysms were evaluated with $5-\mathrm{mm}$ collimation, allowing a $15-\mathrm{cm}$ scan length. The superior extent for each of these studies was at the level of the celiac axis. For purposes of 3D rendering, targeted reconstructions were obtained at $1.5-\mathrm{mm}$ intervals for the renal artery evaluations and at $2.5-\mathrm{mm}$ intervals for the aortic aneurysm evaluations. An image matrix of $512 \times 512$ pixels with a field of view of 22 $\mathrm{cm}$ yielded a pixel size of $0.43 \mathrm{~mm}$ in the transaxial plane.

For the present study, each patient's CT angiogram was rendered into six SSD models by varying the lower segmentation threshold from 80 to $130 \mathrm{HU}$ in increments of $10 \mathrm{HU}$. The upper threshold was maintained at a constant value of $400 \mathrm{HU}$. The resulting images were rotated to reduce (as much as possible) any overlap between the spine and the vascular structures of interest. A line was drawn manually around the spine and ribs to exclude these structures. Subsequently, arterial structures were identified by an algorithm (supplied with the General Electric 3D package) that searches for contiguous voxels within a specified threshold range. Finally, a surface-rendering technique was applied to the resulting volumetric data set to create a $3 \mathrm{D}$ arteriogram.

Hard-copy SSD images were photographed in 24 projections: coronal anteroposterior (AP), coronal posteroanterior (PA), transaxial from cranial, transaxial from caudal, frontal AP rotated $40^{\circ}$ and $60^{\circ}$ cranially, frontal AP rotated $40^{\circ}$ and $60^{\circ}$ caudally, frontal AP rotated by $20^{\circ}, 40^{\circ}, 60^{\circ}$, and $80^{\circ}$ to each the right and the left, frontal AP with $20^{\circ}$ cranial angulation rotated by $20^{\circ}, 40^{\circ}, 60^{\circ}$, and $80^{\circ}$ degrees to each the right and the left. These images were filmed on two sheets of 14- $\times 17$-in film. Multiple projections were imaged to assure that the vessels of interest would be displayed free of overlying tissues. The window and level settings were kept at their default values for SSD images.
Two radiologists (E.J.H. and R.J.W.) blinded to all conventional angiographic results reviewed the six sets of CT angiograms for each patient and selected an appropriate threshold value based upon the following criteria: The threshold should exclude soft tissues overlying the arteries of interest. The threshold should include the main and first-order segmental branches of the renal arteries. When these two criteria conflicted, a threshold was selected (subjectively) to optimize visualization of the arteries and to exclude as much overlying soft tissue as possible.

Each patient had a conventional arteriogram including aortography within 2 weeks of the CT angiogram. Selective injections of the renal arteries were obtained when necessary for further evaluation of the renal arteries. The two radiologists who originally reviewed only the SSD images reviewed these images again together with the conventional arteriograms. The severity of stenosis in each renal artery was evaluated on the frontal and frontal-oblique SSD images at each segmentation threshold, as well as on the conventional arteriogram. Each artery was graded on a scale of 0 to 4 : 0 , normal vessel; 1 , mild stenosis $(<50 \%)$; 2 , moderate stenosis $(50 \%$ to $75 \%)$; 3 , severe stenosis (7699\%); and 4, occlusion. The ideal threshold for each CT angiogram was determined by a comparison of the severity of RAS on SSD images and on the conventional arteriogram. When more than one threshold provided an appropriate classification of the renal arteries, the mean of the thresholds that matched the arteriographic findings was selected. To assess the correlation between the ideal segmentation threshold and the threshold determined by the initial review of the SSD images, a Spearman correlation coefficient was computed.

Measurements of peak enhancement were obtained over regions of interest in the aorta, liver, pancreas, spleen, renal cortex, and renal medulla of each patient. Twelve consecutive transaxial images, centered at the level of the head of the pancreas, were selected for density measurements in each patient. Regions of interest varying in size from 8 to 18 $\mathrm{mm}^{2}$ were placed within the parenchyma of the tissues of interest in each of these 12 images. A mean pixel value was obtained for each region of interest; the peak CT density of each tissue type over all 12 images was recorded. Given that one objective of tissue segmentation is to exclude nonvascular visceral tissues, the lower threshold should increase as a function of visceral enhancement. To quantify the correlation between the ideal segmentation threshold and visceral enhancement, a Spearman correlation coefficient ${ }^{11}$ was computed for each measured visceral structure using Systat 5.0 (Systat Inc, Evanston, IL).

\section{RESULTS}

The appearance of RAS changed as the segmentation threshold was raised from 80 to $130 \mathrm{HU}$. Stenoses appeared discontinuous with higher threshold values (Fig 1). In several instances, entire segmental vessels were eliminated when threshold values were raised (Fig 2).

The segmentation threshold values selected by evaluation of the SSD images alone were 

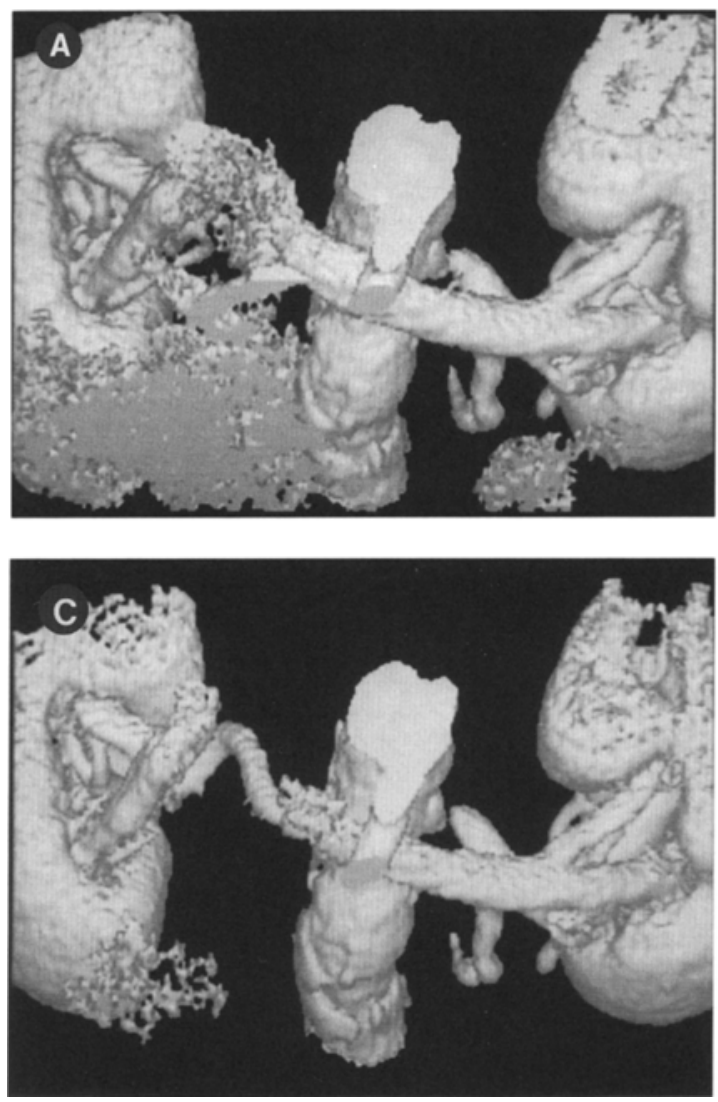
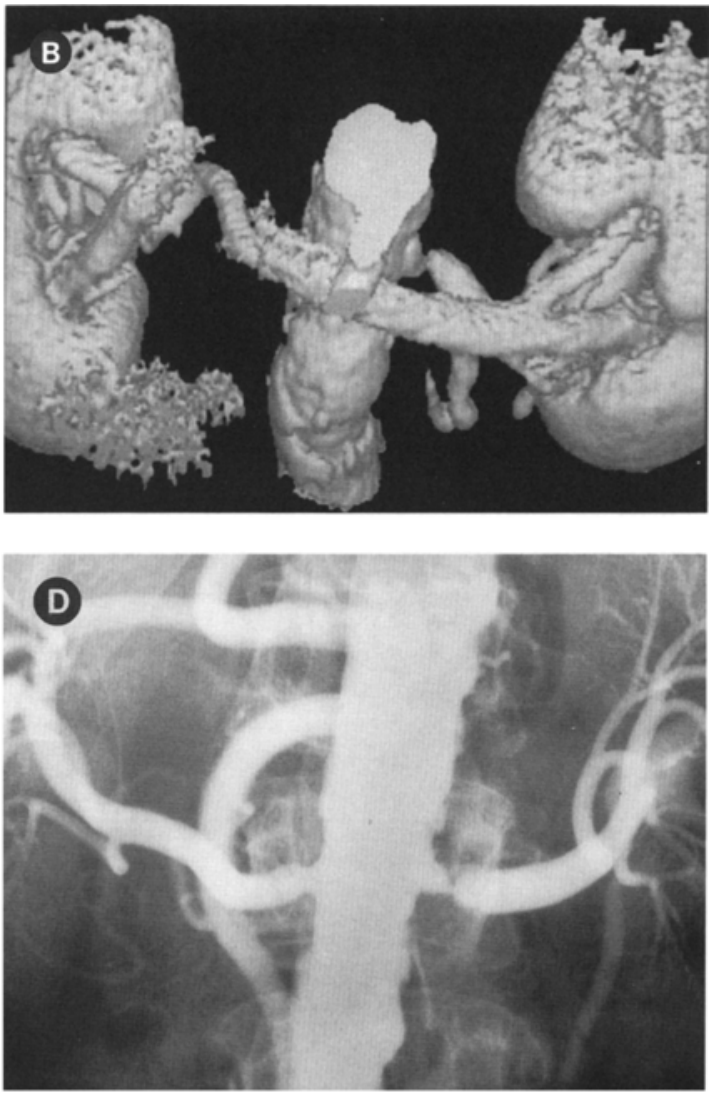

Fig 1. Effect of segmentation threshold on the SSD appearance of a $90 \%$ stenosis at the ostium of the left renal artery. (A) Threshold, 80; (B) threshold, 110; (C) threshold, 120; (D) conventional arteriogram.

relatively close to the ideal values determined by comparison with arteriography (Table 1, $r_{s}=.42$ ). Using these threshold values, the grade of stenosis visualized in the main renal artery by SSD imaging was the same as that visualized on conventional arteriography for the 12 vessels studied with $3-\mathrm{mm}$ collimation. For the vessels imaged with $5-\mathrm{mm}$ collimation, the grade of stenosis determined by SSD imaging differed from the grade shown by conventional arteriography in four of six vessels. None of these discrepancies was explained on the basis of vascular calcifications. Only one of these discrepancies was explained on the basis of an inappropriate segmentation threshold.

All five accessory renal arteries seen on conventional arteriography were also identified by CT angiography. However, artifactual stenoses were suggested in each of these accessory renal arteries (Fig 3), even at the "ideal" SSD thresholds (as determined by the main renal artery appearance on SSD versus conventional arteriography).

The Spearman correlation coefficients $\left(r_{s}\right)$ relating visceral organ enhancement to the ideal segmentation threshold are presented in Table 2. Contrary to our expectations, six of seven tissues showed a negative correlation with the ideal threshold, and the remaining tissue showed only a weak positive correlation of .009 . The lack of a positive correlation between visceral enhancement and the ideal segmentation threshold suggests that visceral enhancement will not provide an accurate predictor of the ideal segmentation threshold.

\section{DISCUSSION}

SSD and MIP are the two rendering techniques most frequently applied to generate angiographic images from transaxial CT scans. SSD creates a 3D image-objects in this image are only visible when they are not covered by 


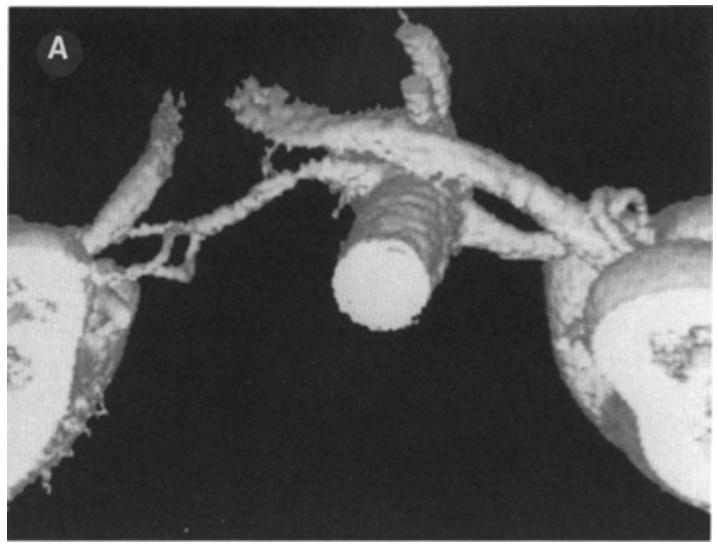

Fig 2. Effect of segmentation threshold on the SSD appearance of normal main and segmental renal arteries. (A) Threshold, 80; (B) threshold, 90; (C) threshold, 100. As the threshold is increased, the right renal artery appears discontinuous and two of the three segmental branches of the right renal artery disappear.

other objects. The sense of depth is enhanced by shading of surfaces to simulate reflection from an imaginary light source. A principle advantage of this technique is the perception of three dimensionality. The principle disadvantage is an inability to distinguish objects with different CT densities (ie, vascular calcification from the enhanced vessel lumen). MIP creates an image that more closely resembles the conventional arteriogram. MIP images are created through a process of ray tracing-multiple rays corresponding to the individual pixels on the display matrix are projected through the image volume; the maximum voxel value traversed by each ray is displayed in the corresponding pixel on the display matrix. A generally recognized advantage of MIP rendering is the ability to distinguish vascular calcifications from the enhanced vessel lumen. ${ }^{3}$ Although a limited form of manual tissue segmentation is usually required to remove osseous structures for MIP rendering, MIP rendering for CT angiography can be performed without a segmentation threshold. Thus, MIP images may not be subject
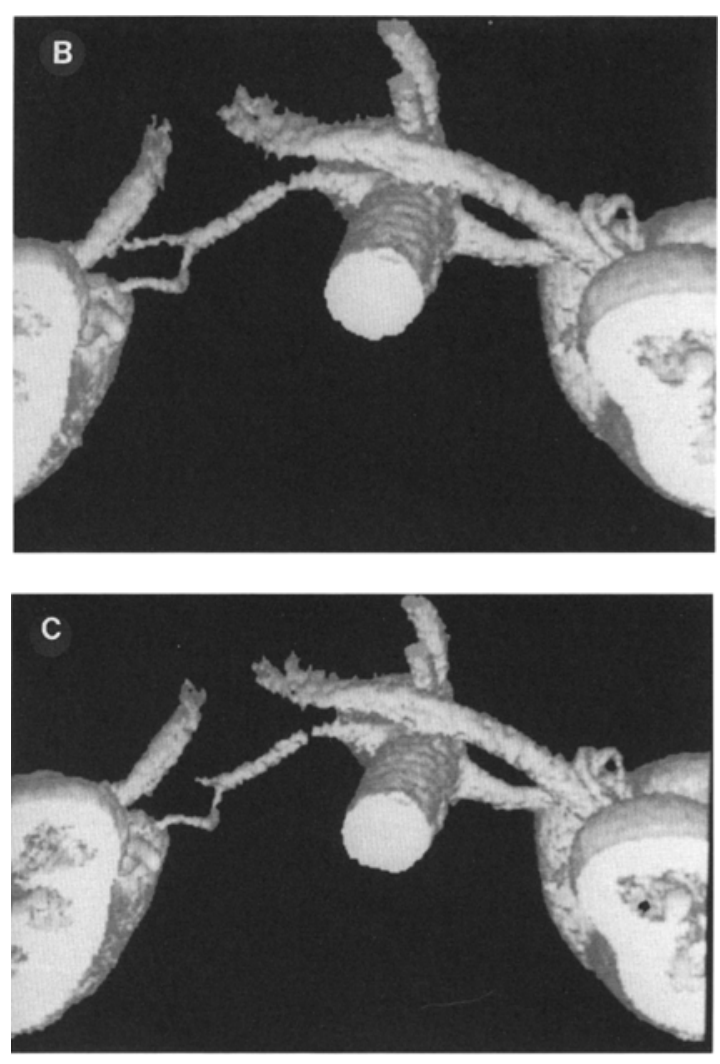

to errors introduced by tissue segmentation thresholds.

The appearance of stenoses and small vessels on SSD images depends upon the segmentation threshold (Figs 1 and 2). Because there are no generally accepted objective criteria for choosing the segmentation threshold, two physicians rendering a SSD CT angiogram from a single data set might visualize different grades of stenosis on the basis of different segmentation thresholds. The present study attempted to define an objective, quantitative criterion for selecting the lower threshold for tissue segmentation. However, the correlation between the ideal threshold as shown by conventional arteriography and measured organ enhancement was no better than the correlation with a threshold selected by subjective evaluation of SSD images. Although only one stenosis was slightly overestimated in our population on the basis of an inappropriate threshold (Table 1, patient no. 3 ), more such errors might be expected in a larger population.

The most important determinant for accurate 
Table 1. Segmentation Thresholds and Visualized Grade of Stenosis

\begin{tabular}{|c|c|c|c|c|c|c|c|c|}
\hline \multirow{2}{*}{$\begin{array}{l}\text { Patient } \\
\text { No. }\end{array}$} & \multirow{2}{*}{$\begin{array}{c}\text { Study } \\
\text { Purpose }\end{array}$} & \multirow{2}{*}{$\begin{array}{c}\text { Ideal } \\
\text { Threshold }\end{array}$} & \multirow{2}{*}{$\begin{array}{l}\text { Subjective } \\
\text { Threshold }\end{array}$} & \multicolumn{2}{|c|}{ Arteriography } & \multicolumn{2}{|c|}{$\begin{array}{c}\text { SSD CT } \\
\text { Angiography }\end{array}$} & \multirow{2}{*}{$\begin{array}{l}\text { Difference } \\
\text { in Grading }\end{array}$} \\
\hline & & & & LRA & RRA & LRA & RRA & \\
\hline \multirow[t]{2}{*}{1} & AAA & 80 & 80 & 1 & & 3 & & 2 \\
\hline & & & & & 4 & & 4 & 0 \\
\hline \multirow[t]{2}{*}{2} & AAA & 100 & 100 & 2 & & 3 & & 1 \\
\hline & & & & & 1 & & 0 & -1 \\
\hline \multirow[t]{2}{*}{3} & AAA & 100 & 110 & 0 & & 0 & & 0 \\
\hline & & & & & 2 & & 3 & 1 \\
\hline \multirow[t]{2}{*}{4} & RAS & 100 & 105 & 3 & & 3 & & 0 \\
\hline & & & & & 0 & & 0 & 0 \\
\hline \multirow[t]{2}{*}{5} & RAS & 110 & 105 & 3 & & 3 & & 0 \\
\hline & & & & & 1 & & 1 & 0 \\
\hline \multirow[t]{2}{*}{6} & RAS & 90 & 110 & 0 & & 0 & & 0 \\
\hline & & & & & 0 & & 0 & 0 \\
\hline \multirow[t]{2}{*}{7} & RAS & 100 & 130 & 0 & & 0 & & 0 \\
\hline & & & & & 0 & & 0 & 0 \\
\hline \multirow[t]{2}{*}{8} & RAS & 120 & 110 & 0 & & 0 & & 0 \\
\hline & & & & & 0 & & 0 & 0 \\
\hline \multirow[t]{2}{*}{9} & RAS & 120 & 125 & 4 & & 4 & & 0 \\
\hline & & & & & 2 & & 2 & 0 \\
\hline
\end{tabular}

For each of the nine subjects, the ideal segmentation threshold was determined by comparison with arteriography, whereas the subjective threshold was determined by evaluation of the SSD images alone. The grade of stenosis ( 0 through 4$)$ for each main renal artery is reported as shown on conventional arteriography and as shown with SSD CT angiography using the subjective threshold.
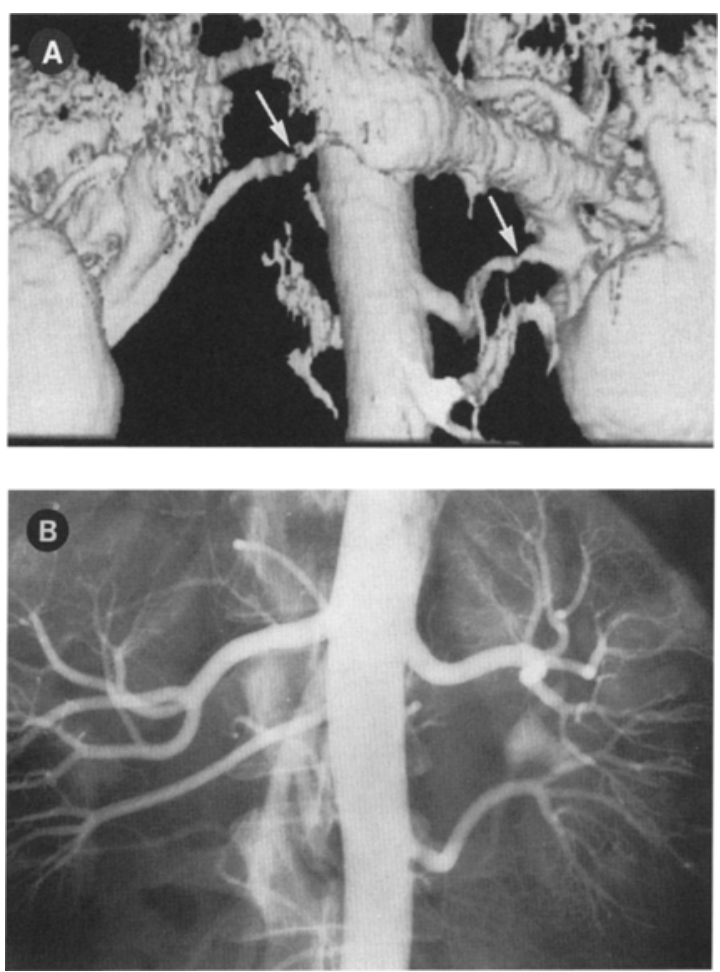

Fig 3. Artifactual stenoses in the SSD display of accessory renal arteries may be related to the lack of sufficient resolution in the Z-axis. (A) SSD image suggests a proximal stenosis in the fower pole accessory artery to the right kidney and a distal beaded appearance in the lower pole accessory artery to the left kidney. (B) Conventional arteriogram shows normal accessory arteries. grading of stenosis in the present study was $\mathrm{Z}$-axis resolution. All errors in the grading of main RAS occurred in patients evaluated for infrarenal aortic aneurysms whose renal arteries were evaluated with 5-mm collimation. Artifactual stenoses were also visualized in five accessory arteries studied with 3-mm collimation. Accessory arteries are generally smaller than the main renal arteries and may not be adequately resolved with $3-\mathrm{mm}$ collimation. Thus, even when the selected segmentation threshold is in agreement with the threshold that might be chosen by comparison with arteri-

Table 2. Tissue Enhancement and Correlation With the Ideal Segmentation Threshold

\begin{tabular}{lcc}
\hline \multicolumn{1}{c}{ Tissue } & $\begin{array}{c}\text { Peak } \\
\text { Enhancement } \\
\text { (HU) (range) }\end{array}$ & $r_{s}$ Value \\
\hline Aorta & $303(208-443)$ & -.079 \\
Renal cortex & $171(91-310)$ & -.052 \\
Renal medulla & $66(31-96)$ & -.044 \\
Pancreas & $113(69-191)$ & -.017 \\
Spleen & $105(53-180)$ & -.289 \\
Liver & $75(32-119)$ & .009 \\
\hline
\end{tabular}

The mean value of peak tissue enhancement and the range are presented for the six tissues studied. Spearman correlation coefficients have been calculated to relate tissue enhancement to the ideal segmentation threshold as shown by comparison with arteriography. A strongly positive correlation is desirable for tissue enhancement to be predictive of segmentation threshold. 
ography, the size of the vessel being imaged relative to the image resolution is an important determinant of accuracy in grading stenosis.

The fundamental problem with grading of stenoses by SSD CT angiography is related to the CT density values of small vessels and areas of stenosis. A recent study has examined the effect of varying scan parameters such as slice collimation upon the appearance of RAS. ${ }^{12}$ When anatomic details of interest are smaller than the CT axial resolution or slice thickness, their CT density will be reduced by partial volume averaging. When the lower threshold for SSD rendering is greater than the CT density of the vessel, that vessel (or portion thereof) is eliminated from the reconstructed image. This problem may be compounded by the lack of consistent objective criteria for selecting the segmentation threshold.

Why was the correlation between organ enhancement and the ideal threshold so poor? Ideally, a CT angiogram would be performed in the first few seconds of the arterial phase of contrast circulation, before any parenchymal enhancement. However, the helical scan requires a finite time interval during which visceral organs enhance at rates that vary by organ and by person. A threshold that is sufficient to exclude one visceral organ may not be sufficient to exclude another. Furthermore, the relationship between vascular enhancement and visceral organ enhancement differs among patients. Thus, it is not surprising that a threshold chosen by subjective evaluation of SSD images for each patient may provide a better approximation of the ideal threshold than a value calculated on the basis of measured visceral enhancement.

Theoretically, MIP rendering will be less affected by reduced $\mathrm{CT}$ density in small vessels because it does not use a lower threshold to exclude structures from the reconstructed image. However, with MIP imaging the window/ level display settings do serve a similar purpose to SSD thresholding. Inappropriate manipulation of these settings might result in poor estimates of the grade of stenosis. A controlled comparison study of MIP rendering at various window/level settings might be useful to show whether this represents a significant problem.

On the basis of our series, it appears that the segmentation threshold for SSD CT imaging should be selected by qualitative evaluation of the resulting SSD images rather than by measurements of tissue enhancement. Using this technique with 3-mm scan collimation, we achieved good correlation with conventional arteriography for grading of stenoses in the main renal artery. However, smaller vessels such as segmental and accessory renal arteries will require narrower collimation for adequate evaluation. Further evaluation is warranted to determine whether inappropriate threshold selection will produce inaccurate grading of stenoses in these smaller vessels.

\section{REFERENCES}

1. Fishman EK, Magid D, Ney DR, et al: Threedimensional imaging. Radiology 181:321-337, 1991

2. Magnusson M, Lenz R, Danielsson PE: Evaluation of methods for shaded surface display of CT volumes. Comput Med Imaging Graph 15:247-256, 1991

3. Napel SA, Marks MA, Rubin GD, et al: CT angiography with spiral CT and maximum intensity projection. Radiology 185:607-610, 1992

4. Farrell EJ, Zappulla RA: Three-dimensional data visualization and biomedical application. CRC Crit Rev Biomed Eng 16:323-363, 1989

5. Rubin GD, Dake MD, Napel SA, et al: Threedimensional spiral CT angiography of the abdomen: Initial clinical experience. Radiology 186:147-152, 1993

6. Rubin GD, Walker DJ, Dake MD, et al: Threedimensional spiral computed tomographic angiography: An alternative imaging modality for the abdominal aorta and its branches. J Vasc Surg 18:656-665, 1993

7. Schwartz RB, Jones KM, Chernoff DM, et al: Com- mon carotid artery bifurcation: Evaluation with spiral CT. Radiology 185:513-519, 1992

8. Ney DR, Fishman EK, Niederhuber JE: Threedimensional display of hepatic venous anatomy generated from spiral computed tomography data: Preliminary results. J Digit Imaging 5:242-245, 1992

9. Galanski M, Prokip M, Chavan A, et al: Renal artery stenosis: Spiral CT angiography. Radiology 189:185-192, 1993

10. Rubin GD, Dake MD, Napel S, et al: Spiral CT of renal artery stenosis: Comparison of three-dimensional rendering techniques. Radiology 190:181-189, 1994

11. Mendenhall W, Scheaffer RL: Non-parametric statistics, in: Mathematical Statistics With Applications. North Scituate, MA, Duxbury, 1973, pp 548-551

12. Brink JA, Lim JT, Wang G, et al: Technical optimization of spiral CT for depiction of renal artery stenosis: In vitro analysis. Radiology 194:157-163, 1995 